\title{
PETTIS INTEGRABILITY AND THE EQUALITY OF THE NORMS OF THE WEAK* INTEGRAL AND THE DUNFORD INTEGRAL
}

\author{
ELIZABETH M. BATOR
}

\begin{abstract}
If $(\Omega, \Sigma, \mu)$ is a perfect finite measure space and $X$ is a Banach space, then it is shown that $X^{*}$ has the $\mu$-Pettis Integral Property if and only if

$$
\|\left(\text { weak }^{*}\right)-\int_{\Omega} f d \mu\|=\|(\text { Dunford })-\int_{\Omega} f d \mu \|
$$
\end{abstract}

for every bounded weakly measurable function $f: \Omega \rightarrow X^{*}$.

1. Introduction. Let $X$ be a Banach space with dual $X^{*}$ and $(\Omega, \Sigma, \mu)$ a finite measure space. If $f: \Omega \rightarrow X^{*}$ is bounded and weakly measurable, that is if $x^{* *} \circ f$ is measurable for every $x^{* *} \in X^{* *}$, then it can easily be shown that

(i) for every $E \in \Sigma$, there exists $x_{E}^{*} \in X^{*}$ such that, for every $x \in X$,

and

$$
x_{E}^{*}(x)=\int_{E} \hat{x} \circ f d \mu
$$

(ii) for every $E \in \Sigma$, there exists $x_{E}^{* * *} \in X^{* * *}$ such that, for every $x^{* *} \in X^{* *}$,

$$
x_{E}^{* * *}\left(x^{* *}\right)=\int_{E} x^{* *} \circ f d \mu .
$$

The element $x_{E}^{*}$ is called the weak ${ }^{*}$ integral of $f$ over $E$, denoted by $\left(\mathrm{w}^{*}\right)-\int_{E} f d \mu$, and $x_{E}^{* * *}$ is called the Dunford integral of $f$ over $E$, denoted (D)- $\int_{E} f d \mu$. By definition, $f$ is Pettis integrable if and only if (D)- $\int_{E} f d \mu \in X^{*}$.

A Banach space $Y$ is said to have the $\mu$-Pettis Integral Property, or $\mu$-PIP if every bounded weakly measurable function $f: \Omega \rightarrow Y$ is Pettis integrable. Characterizations and properties of Pettis integrable functions, spaces with the $\mu$-PIP, and integration of universally weakly measurable functions can be found in $[1,5,6,8$ 14, 16]. Clearly, $X^{*}$ has the $\mu$-PIP if and only if for every $f: \Omega \rightarrow X^{*}$ that is bounded and weakly measurable, $\left(\mathrm{w}^{*}\right)-\int_{E} f d \mu=(\mathrm{D})-\int_{E} f d \mu$ for every $E \in \Sigma$. We show that in fact if $(\Omega, \Sigma, \mu)$ is perfect, then $X^{*}$ has $\mu$-PIP if and only if $\left\|\left(\mathrm{w}^{*}\right)-\int_{\Omega} f d \mu\right\|=$ $\left\|(\mathrm{D})-\int_{\Omega} f d \mu\right\|$ for every such function $f$.

2. Preliminary results. If $A$ is a finite subset of a Banach space $X$ and $\varepsilon>0$, then we define

$$
C_{A, \varepsilon}=\left\{x^{*} \in B^{*}:\left|x^{*}(x)\right|<\varepsilon \text { for every } x \in A\right\},
$$

where $B^{*}=\left\{x^{*} \in X^{*}:\left\|x^{*}\right\| \leqslant 1\right\}$.

Received by the editors November 18, 1984. Presented January 9, 1985 at the annual meeting of the AMS in Anaheim, California.

1980 Mathematics Subject Classification. Primary 28B05, 46G10.

Key words and phrases. Banach space, Pettis integral, Pettis norm, weak measurability.

${ }^{1}$ Partially supported by Faculty Research Grant from North Texas State University.

(1)1985 American Mathematical Society $0002-9939 / 85 \$ 1.00+\$ .25$ per page 
Lemma 1. Let $X$ be a Banach space and $x^{* *} \in X^{* *}$. Suppose for every $\eta>0$ there exists a finite subset $A$ of $X^{*}$ and $\varepsilon>0$ such that if $x^{*} \in C_{A, \varepsilon}$, then $\left|x^{* *}\left(x^{*}\right)\right|<\eta$. Then $x^{* *} \in X$.

Proof. Let $x_{\alpha}^{*}$ be a net in $\frac{1}{2} B^{*}$ such that $x_{\alpha}^{*}$ converges weak ${ }^{*}$ to $x^{*}$. Then $\left(x_{\alpha}^{*}-x^{*}\right) \in B^{*}$ and is eventually in $C_{A, \varepsilon}$ for every $A, \varepsilon$. Hence, $x^{* *}\left(x_{\alpha}-x^{*}\right)$ converges to zero. Consequently, $x^{* *}$ is weak* continuous on $B^{*}$, and hence, $x^{* *} \in X$.

A function $f$ from a measure space $(\Omega, \Sigma, \mu)$ to a dual Banach space $X^{*}$ is weak* measurable if $\hat{x} \circ f$ is measurable for every $x \in X$. If $f$ is weak* measurable we define the Pettis norm of $f$ by

$$
\|f\|_{P}=\sup _{x \in B} \int_{\Omega}|\hat{x} \circ f| d \mu
$$

where $B$ is the closed unit ball of $X$. If $f$ is bounded, we also define the operator $T_{f}$ : $X \rightarrow L^{1}$ by $T_{f}(x)=\hat{x} \circ f$ for $x \in X$. It is clear that the operator norm of $T_{f},\left\|T_{f}\right\|_{\mathrm{oP}}$, is the same as the Pettis norm of $f$.

If $\left(f_{\alpha}\right)_{\alpha \in \Gamma}$ is a net of weak* measurable functions and if $\Sigma_{\alpha}$ is a sub $\sigma$-algebra of $\Sigma$ for every $\alpha \in \Gamma$, then we say $\left(f_{\alpha}, \Sigma_{\alpha}\right)_{\alpha \in \Gamma}$ is a weak* martingale if

(i) $\Sigma_{\alpha} \subset \Sigma_{\beta}$ if $\alpha<B$,

(ii) for every $x \in X, E\left(\hat{x} \circ f_{\beta} \mid \Sigma_{\alpha}\right)=\hat{x} \circ f_{\alpha}$ if $\alpha<\beta$,

where $E\left(\cdot \mid \Sigma_{\alpha}\right)$ is the usual conditional expectation operator with respect to $\Sigma_{\alpha}$ (see [3]). This just says that $\left(\hat{x} \circ f_{\alpha}, \Sigma_{\alpha}\right)_{\alpha \in \Gamma}$ is a scalar-valued martingale for every $x \in X$.

We need the following rather deep result of Fremlin for perfect measure spaces [7]. (See $[7,15]$ for definitions and properties of perfect spaces.)

FREMLIN'S THEOREM. Let $(\Omega, \Sigma, \mu)$ be a finite perfect measure space and $\left(f_{n}\right)_{n=1}^{\infty}$ be a sequence of measurable extended real-valued functions on $\Omega$. Then either $\left(f_{n}\right)_{n=1}^{\infty}$ has a subsequence which converges a.e. or $\left(f_{n}\right)_{n=1}^{\infty}$ has a subsequence having no measurable pointwise cluster points.

We are now able to prove

Proposition 2. Let $(\Omega, \Sigma, \mu)$ be a perfect measure space and $X$ a Banach space. Suppose $f: \Omega \rightarrow X^{*}$ is bounded and weak* measurable. Then the following are equivalent:

(i) $T_{f}: X \rightarrow L^{1}(\mu)$ is a compact operator.

(ii) If $\left(f_{\alpha}, \Sigma_{\alpha}\right)_{\alpha \in \Gamma}$ is any bounded weak* martingale such that $\hat{x} \circ f_{\alpha}$ converges to $\hat{x} \circ f$ in $L^{1}(\mu)$ for every $x \in X$, then $f_{\alpha}$ converges to $f$ in Pettis norm.

(iii) There exists a net of bounded simple functions $\left(f_{\alpha}\right)_{\alpha \in \Gamma}$, such that $f_{\alpha}$ converges to $f$ in Pettis norm.

Proof. Without loss of generality $f$ takes its range in $B^{*}$.

(i) $\Rightarrow$ (ii). Suppose $T_{f}$ is compact and let $\left(f_{\alpha}, \Sigma_{\alpha}\right)_{\alpha \in \Gamma}$ be any bounded weak* martingale such that $\hat{x} \circ f_{\alpha} \rightarrow \hat{x} \circ f$ in $L^{1}(\mu)$. Letting $T_{f_{\alpha}}: X \rightarrow L^{1}(\mu)$ by $T_{f_{\alpha}}(x)=$ $\hat{x} \circ f_{\alpha}$, we note that $T_{f_{\alpha}}$ converges to $T_{f}(x)$ in $L^{1}(\mu)$ and $T_{f_{\alpha}}(x)=E\left(\hat{x} \circ f \mid \Sigma_{\alpha}\right)$. It 
suffices to show that $T_{f_{\alpha}}(x)$ converges to $T_{f}(x)$ uniformly on $B$, as this says

$$
\begin{aligned}
& \lim _{\alpha} \sup _{x \in B}\left\|T_{f_{\alpha}}(x)-T_{f}(x)\right\|_{L^{1}}=0 \text {, or } \\
& \lim _{\alpha} \sup _{x \in B} \int_{\Omega}\left|\hat{x} \circ f_{\alpha}-\hat{x} \circ f\right| d \mu=0 .
\end{aligned}
$$

Let $\varepsilon>0$. Since $T_{f}$ is compact, there exists $x_{1}, \ldots, x_{n} \in B$ such that $T_{f}(B) \subset$ $\bigcup_{i=1}^{n}\left\{g:\left\|g-T_{f}\left(x_{i}\right)\right\|_{L^{1}}<\varepsilon / 3\right\}$. Choose $\beta$ such that if $\alpha>\beta$ then

$$
\left\|T_{f_{\alpha}}\left(x_{i}\right)-T_{f}\left(x_{i}\right)\right\|_{L^{1}}<\varepsilon / 3
$$

for $i=1, \ldots, n$. Let $x \in B$ and let $x_{i}$ be such that $\left\|T_{f}(x)-T_{f}\left(x_{i}\right)\right\|<\varepsilon / 3$. We note that $E\left(\cdot \mid \Sigma_{\alpha}\right)$ is an $L^{1}$ contraction [3]. Then if $\alpha>\beta$,

$$
\begin{aligned}
& \left\|T_{f_{\alpha}}(x)-T_{f}(x)\right\|_{L^{1}} \\
& \quad \leqslant\left\|T_{f_{\alpha}}(x)-T_{f_{\alpha}}\left(x_{i}\right)\right\|_{L^{1}}+\left\|T_{f_{\alpha}}\left(x_{i}\right)-T_{f}\left(x_{i}\right)\right\|_{L^{1}}+\left\|T_{f}\left(x_{i}\right)-T_{f}(x)\right\|_{L^{1}}<\varepsilon .
\end{aligned}
$$

(ii) $\Rightarrow$ (iii). It suffices to show that there exists a bounded weak* martingale $\left(f_{\alpha}, \Sigma_{\alpha}\right)_{\alpha \in \Gamma}$ such that $f_{\alpha}$ is simple for every $\alpha \in \Gamma$, and $\hat{x} \circ f_{\alpha} \rightarrow \hat{x} \circ f$ in $L^{1}$ for every $x \in X$.

Let $\Pi$ be the set of finite partitions of $\Omega$ into elements of $\Sigma$ directed by refinement. If $\pi \in \Pi$, let $\Sigma_{\pi}$ be the finite $\sigma$-algebra generated by the elements of $\pi$ and let

$$
f_{\pi}=\sum_{A \in \pi} \frac{\left(\mathrm{w}^{*}\right)-\int_{A} f d \mu}{\mu(A)} \chi_{A} .
$$

It is clear that $\left(f_{\pi}, \Sigma_{\pi}\right)_{\pi \in \Pi}$ is a weak* martingale, each $f_{\pi}$ is simple and the fact that $\hat{x} \circ f_{\pi} \rightarrow \hat{x} \circ f$ in $L^{1}(\mu)$ follows from scalar-valued martingale convergence theorems [3].

(iii) $\Rightarrow$ (i). Suppose $\left(f_{\alpha}\right)_{\alpha \in \Gamma}$ is a net of simple functions converging to $f$ in Pettis norm. Then $T_{f_{\alpha}}$ converges to $T_{f}$ in operator norm. Since $T_{f_{\alpha}}$ is a finite rank operator for each $\alpha, T_{f}$ is compact.

The following was first observed by Stegall [8].

Proposition 3. If $(\Omega, \Sigma, \mu)$ is a perfect finite measure space and $f: \Omega \rightarrow X^{*}$ is bounded and weakly measurable, then $T_{f}: X \rightarrow L^{1}$ is compact. Hence there exists a net of simple functions converging to $f$ in Pettis norm.

Proof. Let $\left(x_{n}\right)_{n=1}^{\infty}$ be bounded in $X$. Suppose $\left(\hat{x}_{n} \circ f\right)_{n=1}^{\infty}$ does not have an a.e. convergent subsequence. By Fremlin's theorem, there is a subsequence $\left(\hat{x}_{n,} \circ f\right)_{j=1}^{\infty}$ having no measurable pointwise cluster points. Let $x^{* *}$ be a weak* cluster point of $\left(\hat{x}_{n_{j}}\right)_{j=1}^{\infty}$ in $X^{* *}$. Hence $x^{* *} \circ f$ is a pointwise cluster point of $\left(\hat{x}_{n_{j}} \circ f\right)_{j=1}^{\infty}$ and is therefore nonmeasurable. This contradicts the weak measurability of $f$. Hence some subsequence must converge a.e. and by boundedness this subsequence must converge in $L^{1}(\mu)$. 
3. Main result. Putting together the pieces from $\S 2$ yields

THEOREM 4. If $(\Omega, \Sigma, \mu)$ is a perfect measure space and $X$ is a Banach space, then $X^{*}$ has $\mu$-PIP if and only if for every $f: \Omega \rightarrow X^{*}$ that is bounded and weakly measurable

$$
\left\|\left(\mathrm{w}^{*}\right)-\int_{\Omega} f d \mu\right\|=\left\|(\mathrm{D})-\int_{\Omega} f d \mu\right\| .
$$

In fact if $f$ is not Pettis integrable, then for some $E \in \Sigma$ and $\alpha>0$ there exists a sequence of simple functions $\left(f_{n}\right)_{n=1}^{\infty}$ such that

$$
\left\|\left(\mathrm{w}^{*}\right)-\int_{E} f-f_{n} d \mu\right\| \rightarrow 0 \text { but }\left\|(\mathrm{D})-\int_{E} f-f_{n} d \mu\right\|>\alpha \text { for every } n .
$$

Proof. Of course if $f$ is Pettis integrable then these two norms are the same. Conversely, let $f: \Omega \rightarrow X^{*}$ be bounded and weakly measurable. Without loss of generality, $f$ takes its range in $B^{*}$. By Lemma 1 , it suffices to show that for every $E \in \Sigma$ and $\eta>0$ there exists a finite subset $A$ of $X^{*}$ and $\varepsilon>0$ such that $\left|\int_{E} x^{* *} \circ f d \mu\right|<\eta$ whenever $x^{* *} \in C_{A, \varepsilon}$.

Let $E \in \Sigma$ and $\eta>0$. Let $f_{E}=f \chi_{E}$. Choose by Proposition 3 a simple function $h$ such that $\left\|f_{E}-h\right\|_{P}<n / 2$. Note then by hypothesis

$$
\left\|(\mathrm{D})-\int_{\Omega}\left(f_{E}-h\right) d \mu\right\|=\left\|\left(\mathrm{w}^{*}\right)-\int_{\Omega}\left(f_{E}-h\right) d \mu\right\| \leqslant\left\|f_{E}-h\right\|_{P}<\eta / 2 .
$$

Hence letting $A$ be the range of $h$ and $\varepsilon=\eta / 2$, we see that if $x^{* *} \in C_{A, \varepsilon}$, then

$$
\begin{aligned}
\left|\int_{E} x^{* * \circ} \circ d \mu\right| & \leqslant\left|\int_{\Omega} x^{* *} \circ\left(f_{E}-h\right) d \mu\right|+\left|\int_{\Omega} x^{* *} \circ h d \mu\right| \\
& <\|(\mathrm{D})-\int_{\Omega}\left(f_{E}-h\right) d \mu \mid+\eta / 2<\eta
\end{aligned}
$$

This proves the first assertion. To prove the second, we know that if $f$ is bounded and weakly measurable, we can always find a sequence of simple functions $\left(f_{n}\right)$ such that $\left\|\left(\mathrm{w}^{*}\right)-\int_{\Omega}\left(f-f_{n}\right)\right\|$ converges to zero. If there did not exist an $\alpha>0$ such that $\left\|(\mathrm{D})-\int_{\Omega}\left(f-f_{n}\right)\right\|>\alpha$, then this would force $f$ to be Pettis integrable as in the above argument.

The following example is due to Phillips and is discussed in detail by Geitz in [ 9 and 10]. Let $(\Omega, \Sigma, \mu)$ be usual Lebesgue measure space and $l^{\infty}[0,1]$ be the space of bounded functions with usual supremum norm. Sierpiński constructed a subset $B$ of $[0,1] \times[0,1]$ such that

(i) for every $t_{0} \in[0,1],\left\{s:\left(s, t_{0}\right) \in B\right\}$ is countable, and

(ii) for every $s_{0} \in[0,1],\left\{t:\left(s_{0}, t\right) \notin B\right\}$ is countable.

It is shown in [10] that the function $f:[0,1] \rightarrow l^{\infty}[0,1]$ given by $[f(s)](t)=\chi_{B}(s, t)$ is bounded and weakly measurable but not Pettis integrable with respect to $(\Omega, \Sigma, \mu)$.

It is also shown that if $e_{t_{0}}$ is the evaluation functional at $t_{0}$ on $l^{\infty}[0,1]$, then from (i) we have

$$
\int_{[0,1]} e_{t_{0}} f(s) d \mu(s)=\int_{[0,1]} \chi_{B}\left(s, t_{0}\right) d \mu(s)=0
$$


Hence, $\left\|\left(\mathrm{w}^{*}\right)-\int_{E} f d \mu\right\|=0$ for every $E \in \Sigma$. However, if $\beta \in \mathrm{ba}[0,1]=\left(l^{\infty}[0,1]\right)^{*}$ is such that $\beta$ vanishes on countable sets and $\|\beta\|=1$, then we have by (ii) that, for every $s_{0} \in[0,1]$,

$$
\int_{[0,1]} f\left(s_{0}\right) d \beta=1
$$

Hence, $\int_{E} \int_{[0,1]} f(s) d \beta d \mu(s)=\mu(E)$ for every $E \in \Sigma$, and thus $\left\|(\mathrm{D})-\int_{E} f d \mu\right\|=$ $\mu(E)$ for every $E \in \Sigma$.

4. Observations and questions. Suppose $f: \Omega \rightarrow X^{*}$ is bounded and weak* measurable, that is $\hat{x} \circ f$ is measurable for every $x \in X$, such that $T_{f}$ is weak compact. Hence, $T_{f}^{* *}: X^{* *} \rightarrow L^{1}(\mu)$. This will certainly, but not necessarily, be the case if $f$ is weakly measurable. Since $\left\langle T_{f}^{* *}\left(x^{* *}\right), g\right\rangle=x^{* *}\left(\left(\mathrm{w}^{*}\right)-\int_{\Omega} f g\right)$ for every $g \in L^{\infty}(\mu)$, there exists a function $h_{x^{* *}} \in L^{1}(\mu)$ such that $x^{* *}\left(\left(\mathrm{w}^{*}\right)-\int_{\Omega} f g d \mu\right)=\int_{\Omega} h_{x^{* *}} g d \mu$ for every $g \in L^{\infty}(\mu)$. Note that if $\left(x_{\beta}\right)$ is a net in $X$ such that $\hat{x}_{\beta}$ converges weak* to $x^{* *}$, then clearly $x^{* *} \circ f$ is a pointwise limit of $\left(\hat{x}_{\beta} \circ f\right)$, whereas $h_{x^{* *}}$ is an $L^{1}(\mu)$ limit of $\left(\hat{x}_{\beta} \circ f\right)$. Consequently, for every $x^{* *}$ there exists a sequence $\left(x_{n}\right)_{n=1}^{\infty}$ in $X$ such that $\hat{x}_{n} \circ f$ converges a.e. to $h_{x^{* * *}}$. If $f$ is Pettis integrable, however, it must be the case that $x^{* *} \circ f=h_{x^{* *}}$ a.e. Hence we get

THEOREM 5. Let $X$ be a Banach space and $(\Omega, \Sigma, \mu)$ a finite measure space. Suppose $f: \Omega \rightarrow X^{*}$ is bounded and weakly measurable. Then $f$ is Pettis integrable if and only if, for every $x^{* *} \in X^{* *}$, there exists a bounded sequence $\left(x_{n}\right)_{n=1}^{\infty}$ in $X$ such that both of the following hold:

(i) $\hat{x}_{n} \circ f$ converges a.e. to $x * * \circ f$,

(ii) $\hat{x}_{n}\left(\left(\mathrm{w}^{*}\right)-\int_{E} f d \mu\right)$ converges to $x^{* *}\left(\left(\mathrm{w}^{*}\right)-\int_{E} f d \mu\right)$ for every $E \in \Sigma$.

We observe that condition (i) above guarantees that $\left\|\left(\mathrm{w}^{*}\right)-\int f d \mu\right\|=\left\|(\mathrm{D})-\int f d \mu\right\|$. Hence we get the following corollary from Theorems 4 and 5:

COROllaRY 6. Let $X$ be a Banach space and $(\Omega, \Sigma, \mu)$ a perfect finite measure space. Then $X^{*}$ has $\mu-P I P$ if and only if whenever $f: \Omega \rightarrow X^{*}$ is bounded and weakly measurable then, for every $x^{* *} \in X^{* *}$, there is a bounded sequence $\left(x_{n}\right)_{n=1}^{\infty}$ in $X$ such that $\hat{x}_{n} \circ f$ converges to $x^{* *} \circ f$ a.e.

Question. Is it possible to remove condition (ii) in Theorem 5?

\section{REFERENCES}

1. K. T. Andrews, Universal Pettis integrability, Preprint 1983.

2. E. M. Bator, Duals of separable Banach spaces, $\mathrm{Ph}$. D. Thesis, Pennsylvania State University, 1983.

3. J. Diestel and J. J. Uhl, Jr., Vector measures, Math. Surveys, No. 15, Amer. Math. Soc., Providence, R.I., 1977.

4. N. Dunford and J. T. Schwartz, Linear operators, Part I, Interscience, New York, 1958.

5. G. A. Edgar, Measurability in a Banach space. I, Indiana Univ. Math. J. 26 (1976), 663-677.

6. ___ Measurability in a Banach space. II, Indiana Univ. Math. J. 28 (1979), 559-580.

7. D. H. Fremlin, Pointwise compact sets of measurable functions, Manuscripta Math. 15 (1975), 219-242.

8. D. H. Fremlin and M. Talagrand, A decomposition theorem for additive set functions, with applications to Pettis integrals and ergodic means, Math. Z. 168 (1979), 177-242. 
9. R. F. Geitz, Geometry and the Pettis integral, Trans. Amer. Math. Soc. 269 (1982), 535-548. 10. Pettis integration, Proc. Amer. Math. Soc. 82 (1981), 81-86.

11. R. E. Huff, Remarks on Pettis integration, Preprint 1984.

12. L. H. Riddle and E. Saab, On functions that are universally Pettis integrable, Preprint 1983.

13. L. H. Riddle, E. Saab and J. J. Uhl, Jr., Sets with the weak Radon-Nikodym property in dual Banach spaces, Indiana Univ. Math. J. 32 (1983), 527-541.

14. L. H. Riddle and J. J. Uhl, Jr., Martingales and the fine line between Asplund spaces and spaces not containing a copy of $e_{1}$, Proceedings, Martingale Theory in Harmonic Analysis and Banach Spaces, Cleveland, 1981 (J. A. Chao and W. A. Woyczynski, eds.), Lecture Notes in Math., vol. 939, SpringerVerlag, Berlin and New York.

15. V. V. Sazonov, On perfect measures, Amer. Math. Soc. Transl. (2) 48 (1965), 229-254

16. D. Sentilles and R. F. Wheeler, Pettis integration via the Stonian transform, Pacific J. Math. 107 (1983), 473-496.

Department of Mathematics, North Texas State University, Denton, Texas 76203 